\title{
Calidad biológica y fisicoquímica de tres fuentes de agua y su relación con el fenómeno de El Niño y La Niña
}

\section{Biological and physicochemical quality from three water sources and its relation to the El Niño and La Niña phenomenon}

\author{
Yimmy Montoya-Moreno; Erica Yaneth Patiño Zapata²; Elizabeth Ramírez Arango ${ }^{3}$; Ernesto Raúl Yepes Osorio ${ }^{4}$
}

\begin{abstract}
'Biólogo, M.Sc, Ph.D. Universidad Pontificia Bolivariana, Facultad de Ingeniería. Medellín - Antioquia, Colombia; e-mail: yimmymontoya3@hotmail.com; (Dhttps://orcid.org/0000-0002-1054-279X

Licenciada en Básica primaria, M.Sc. Universidad Pontificia Bolivariana, Facultad de Ingeniería. Medellín - Antioquia, Colombia; e-mail: ericapz@hotmail. com; Dhttps://orcid.org/0000-0002-7355-8764
\end{abstract}

${ }^{3}$ Licenciada en Ciencias Naturales, M.Sc. Universidad Pontificia Bolivariana, Facultad de Ingeniería. Medellín - Antioquia, Colombia; e-mail: astroera@hotmail. com; Dhttps://orcid.org/0000-0001-5096-9571

${ }^{4}$ Licenciado en matemáticas, M.Sc. en Ciencias Naturales y Matemáticas. Universidad Pontificia Bolivariana, Facultad de Ingeniería. Medellín - Antioquia, Colombia; e-mail: ernestoraulyepes@gmail.com; (Dhttps://orcid.org/0000-0002-5112-8034

Cómo citar: Montoya-Moreno, Y.; Patiño Zapata, E.Y.; Ramírez Arango, E.; Yepes Osorio, E.R. 2019. Calidad biológica y fisicoquímica de tres fuentes de agua y su relación con el fenómeno de El Niño y La Niña. Rev. U.D.C.A Act. \& Div. Cient. 22(2):e1328. https://doi. org/10.31910/rudca.v22.n2.2019.1328

Artículo de acceso abierto publicado por Revista U.D.C.A Actualidad \& Divulgación Científica, bajo una licencia Creative Commons CC BY-NC 4.0

Recibido: Abril 22 de 2018

Aceptado: Agosto 24 de 2019

Editado por: Ingeborg Zenner de Polanía

\section{RESUMEN}

En el municipio de El Carmen de Viboral, Antioquia, la cuenca de la quebrada La Cimarronas y sus afluentes Los Andes y La Madera son las principales fuentes de abastecimiento del acueducto de la localidad. Además, en estas y en otras cinco estaciones de muestreo, ubicadas en la parte alta de la microcuenca y sus afluentes, se realizó la evaluación fisicoquímica, microbiológica y biológica, mediante el uso de los macroinvertebrados acuáticos, en tres periodos del ciclo hidrológico de El Niño (2016), los cuales, fueron comparados con tres muestreos del ciclo de La Niña (2011). Se pudo identificar la capacidad de resiliencia ambiental bajo un déficit y exceso de precipitaciones del sistema acuático. Durante el ciclo afectado por El Niño, se presentó mayor concentración de oxígeno disuelto, $\mathrm{pH}$ circumneutral, menor temperatura del agua y mayores concentraciones de nitritos, nitrógeno amoniacal y coliformes totales. Durante el ciclo afectado por La Niña, se manifestó mayor temperatura del agua, sólidos totales, dureza, nitratos, fósforo total y mejores condiciones de calidad biológica del agua, según el índice BMWP. No se registraron variaciones estadísticamente significativas del caudal, la temperatura del agua, la conductividad eléctrica y los índices comunitarios (diversidad, equidad, dominancia y riqueza).

Palabras clave: BMWP; enos; cambio climático; quebradas andinas; macroinvertebrados acuáticos. 


\section{ABSTRACT}

In the municipality of El Carmen de Viboral, Antioquia, the basin of the La Cimarronas stream and its tributaries Los Andes and La Madera, are the main sources of supply of the local aqueduct. In addition, of these, other five sampling stations located in the upper part of the basin and its tributaries, the physico-chemical, microbiological and biological evaluation was carried out through the use of aquatic macroinvertebrates in three periods of the El Niño hydrological cycle (2016), which were compared with three samplings of the La Niña cycle (2011). The capacity of environmental resilience could be identified under a deficit and excess precipitation of the aquatic system. During the cycle affected by El Niño there was a higher concentration of dissolved oxygen, circumneutral $\mathrm{pH}$, lower water temperature and higher concentrations of nitrites, ammonia nitrogen and total coliforms. During the cycle affected by La Niña, there was a higher water temperature, total solids, hardness, nitrates, total phosphorus and better conditions of biological water quality according to the BMWP index. There were no statistically significant variations in flow, water temperature, electrical conductivity and community indices (diversity, equity, dominance and richness).

Keywords: BMWP; Enso; climate change; Andean streams; aquatic macroinvertebrates.

\section{INTRODUCCIÓN}

El agua es un compuesto indispensable para la existencia de vida en el planeta y, además, constituye una fuente importante de sostenibilidad económica y social para la humanidad. Ante una inminente escasez, algunas naciones del mundo han emprendido políticas ambientales para salvaguardar la disponibilidad y la calidad del agua. Es el caso de los países europeos, en donde estudios realizados demuestran que el cambio climático ha aumentado el número de episodios de sequía en ciertas regiones del continente e inundaciones en otras (Comisión Europea, 2000). Childress (2015) habla de la amplia brecha internacional que hay en materia de calidad del agua, entre países desarrollados y subdesarrollados, en donde los pobladores carecen de condiciones mínimas de salubridad, generadas por problemas sociales, como el conflicto, la pobreza, la política internacional, el calentamiento global y la economía mundial. A nivel de Suramérica, Ramírez \& Gutiérrez-Fonseca (2014) realizaron una revisión entre el 2000 y 2014 de todos los trabajos adelantados sobre macroinvertebrados acuáticos, en los que predominan los brasileros (35\%) y colombianos $(17,4 \%)$; no obstante, se plantea que, a largo plazo, se espera que se realicen investigaciones sobre esta comunidad y su relación con el fenómeno de El Niño-La Niña.

El Reporte Nacional del Agua realizado por el IDEAM, en el 2014, revela conclusiones sobre las condiciones actuales del recurso hídrico en Colombia, evidenciando problemáticas, como vulnerabilidad por desabastecimiento y variabilidad climática en 110 municipios, con una población estimada de 17.500 .000 habitantes. También, la afectación a la calidad del agua, por cargas contaminantes de material biodegradable, no biodegradable, nutrientes, metales pesados y mercurio, que se concentra en cerca de 150 municipios y una dependencia alta de agua en los sectores agrícola y pecuario, lo que hace que estos sectores económicos sean vulnerables al cambio climático (IDEAM, 2015). Este diagnóstico nacional refleja que el cambio climático incidirá de manera vertiginosa en la disponibilidad y abastecimiento del agua en el país.

Rocha-Felices (2002) describe que la historia de eventos de "El Niño" ocurre desde 1578, con una regularidad de cada 2 a 7 años y una duración entre 4 a 12 meses, con algunos casos, de hasta 18 meses. Este fenómeno, en sus periodos más intensos, causa desequilibrios y modificaciones en la circulación de las aguas en los océanos y en las corrientes atmosféricas, lo que afecta a todo el globo terráqueo, hacia los cinturones tropical y subtropical de la esfera terrestre, los cuales, benefician e incrementan la producción de precipitaciones torrenciales, dando origen a crecientes, inundaciones, avalanchas, tormentas y ciclones tropicales; de la misma manera, se producen sequías, que dejan como consecuencia catástrofes en todo el planeta (Puerta \& Carvajal, 2008).

Se ha encontrado en diferentes investigaciones que los fenómenos macro climáticos, como El Niño y La Niña-Oscilación del Sur (ENOS), la Oscilación del Sur (OS), la Oscilación del Atlántico Norte (NAO) y la Oscilación Cuasi Bienal (OCB) exhiben una alta influencia en la hidroclimatología colombiana (Aguirre-Cárdenas et al. 2018). El Niño y La Niña, se consideran las fases extremas del fenómeno oceanoatmosférico, conocido como El Niño-Oscilación del Sur, ENSO. Ambas fases, se encuentran asociadas a las anomalías hidrológicas que ocurren en el trópico suramericano, entre otras regiones, básicamente, en escalas de tiempo, que van desde la mensual hasta la interanual (Poveda, 2004).

En Colombia, se han realizado pocas investigaciones que indagan sobre las relaciones entre Enso y los ecosistemas acuáticos, entre las que se encuentran dos tendencias: 1) Relaciones entre el Enso y la precipitación y caudales. En esta línea, Ávila-Díaz et al. (2014) estudiaron la influencia de la oscilación del Pacífico en la oferta hídrica mensual de la cuenca del río Cali, donde encontraron que la oferta hídrica decrece en un $40 \%$ en épocas del fenómeno El Niño y aumenta su disponibilidad, hasta un $65 \%$, en épocas del fenómeno La Niña; también registraron mayores correlaciones entre el Enso y el caudal que con la precipitación. Ramírez-Buíles \& JaramilloRobledo (2009) encontraron en la zona andina central que los meses, históricamente conocidos como los de baja precipitación (diciembre, enero, junio, julio, agosto y septiembre), que no tienen influencia de la zona de interconvergencia tropical son los que muestran una relación estadísticamente y presentaron los mayores coeficientes de correlación entre la lluvia mensual y la temperatura superficial del océano Pacífico, representado en el índice oceánico del Niño. 2) Relación con la variación de la fisicoquímica del agua y el Enso. Sobre este tema, se encuentra menor número de trabajos, entre los que se resalta el de Blanco et al. (2003), quienes evidenciaron que durante El Niño se redujo la precipitación, generando los menores caudales mínimos históricos, pero no alteró la frecuencia de las crecientes. Durante La Niña, el aumento de la precipitación incrementó el caudal promedio y las crecientes tuvieron mayor 
magnitud. La temperatura disminuyó significativamente durante La Niña, aunque las concentraciones de fosfatos, de hierro y de nitratos también aumentaron. En el caso de los nitratos, se asume el incremento como producto de la nitrificación, lo cual, redujo el porcentaje de saturación de oxígeno disuelto. Los incrementos de las anteriores variables dependen de la escorrentía sostenida por periodos mayores a las variaciones del caudal. También, se registró que la turbiedad fue la única variable que aumentó instantáneamente con las lluvias.

Las evidencias de la contaminación se pueden establecer a través del estudio de bioindicadores, dado que permiten monitorear y valorar cambios en el estado del agua. El índice BMWP/Col ha sido el indicador biológico de calidad del agua más usado en Colombia y, actualmente, no se encuentra información disponible sobre cómo varia con relación a los Enos de El Niño y La Niña. Esta investigación parte de la hipótesis de que el fenómeno de El Niño genera reducción del volumen de agua que fluye por las quebradas, debido a la disminución de las precipitaciones, por lo que se espera que se presente una reducción de la calidad biológica y fisicoquímica del agua, a nivel espacial y temporal. En contraste, al comparar los resultados obtenidos con los muestreos realizados por García et al. (2012) durante el fenómeno de La Niña, se espera poder identificar los rangos de variación de los indicadores biológicos y fisicoquímicos.

\section{MATERIALES Y MÉTODOS}

Área de estudio. El municipio de El Carmen de Viboral, está situado al suroriente del departamento de Antioquia, en la subregión Valle de San Nicolás, con coordenadas geográficas de 6 $05^{\circ} 09^{\prime \prime}$ de latitud Norte y 75²0'19' longitud Oeste, a una altura de $2.150 \mathrm{~m}$ s.n.m., con una temperatura media de $17^{\circ} \mathrm{C}$ y un área total de $440 \mathrm{~km}^{2}$ (Figura 1). La principal fuente hídrica para el abastecimiento del acueducto municipal es la cuenca La Cimarronas, que está ubicada en su totalidad dentro del municipio. La temperatura promedio multianual es de $14^{\circ} \mathrm{C}$ y la nubosidad es abundante durante casi todo el año (CORNARE, 2006).

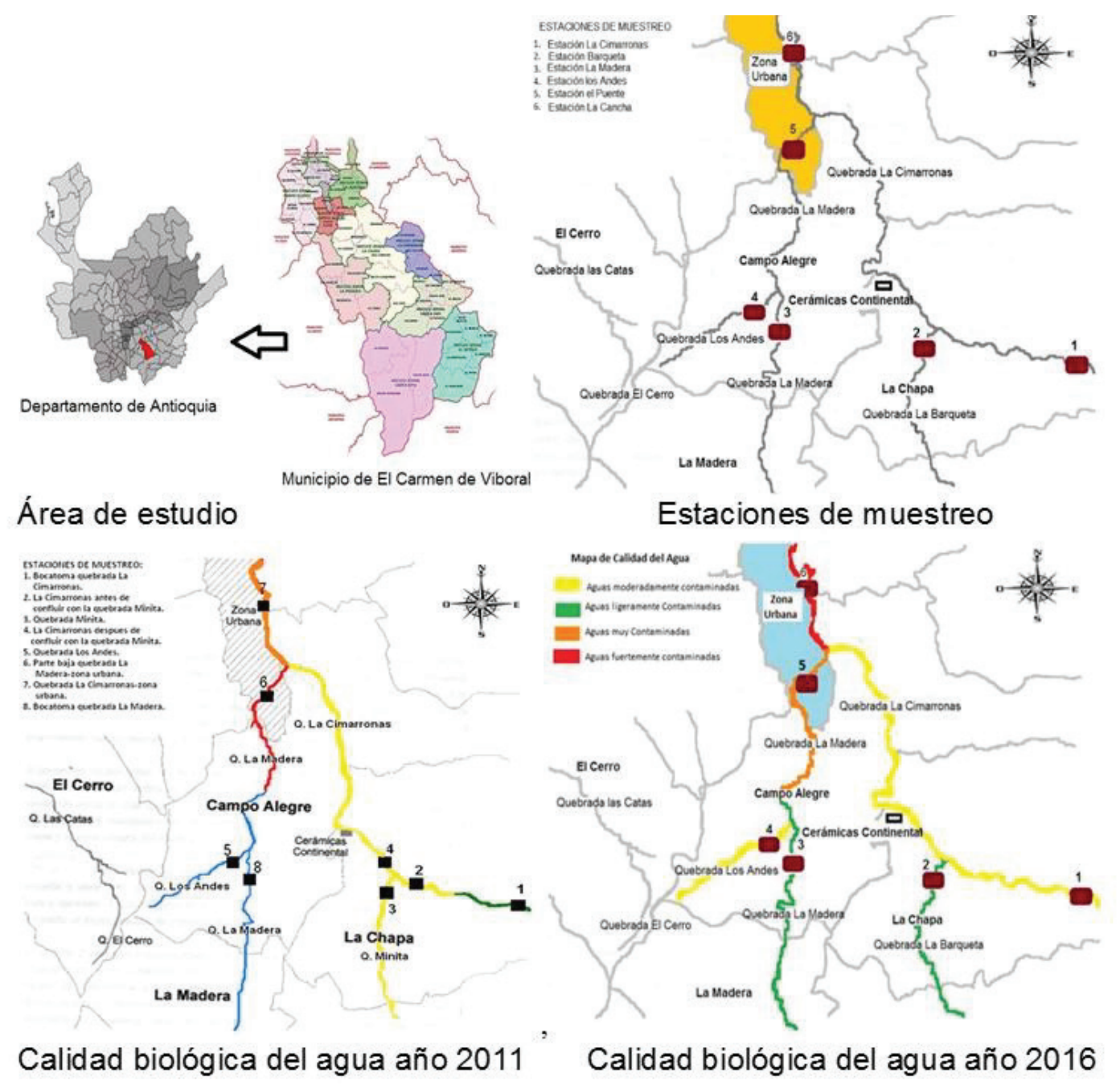

Figura 1. Ubicación del municipio de El Carmen de Viboral, las quebradas y las estaciones de muestreo y el mapa de calidad del agua de 2011-La Niña (García et al. 2012) y de 2016-El Niño (modificado de García et al. 2012). 
Muestreos. Se ubicaron y marcaron seis estaciones de monitoreo, tres, situadas en las quebradas La Barqueta (E2), La Madera (E3) y Los Andes (E4), las cuales, son tributarias de la quebrada La Cimarronas; en esta última, se ubicaron las tres estaciones restantes (E1 zona de la bocatoma, E5 puente lavadero y E6 cancha municipal). En el 2016, se realizaron tres muestreos en las seis estaciones, uno correspondiente al periodo seco (febrero), otro al periodo de transición de época seca a húmeda (abril) y, por último, un muestreo que corresponde al periodo húmedo (junio); esta clasificación pluvial, se definió con base en los promedios históricos de precipitación de la región.

Variables ambientales: Por medio del IDEAM y la empresa La Cimarrona E.S.P., se obtuvieron datos históricos de temperatura del aire, humedad relativa, velocidad de viento, caudales, pluviosidad. La información del ciclo influenciado por el fenómeno de La Niña, se tomó de García et al. (2012).

Variables físicas, químicas y microbiológicas: En cada sitio de muestreo, se evaluaron variables físicas y químicas in situ, como temperatura del agua $\left({ }^{\circ} \mathrm{C}\right)$, oxígeno disuelto $(\mathrm{mg} / \mathrm{l})$, porcentaje de saturación de oxígeno (\%), conductividad eléctrica $(\mu \mathrm{S} / \mathrm{cm}), \mathrm{pH}$ y el caudal. Una muestra de agua de 1 litro fue enviada al laboratorio de CORNARE, donde se utilizó para la determinación de sólidos totales (ST), turbiedad del agua, amonio $\left(\mathrm{N}^{-\mathrm{NH}_{4}}{ }^{+}\right)$, nitritos $\left(\mathrm{N}-\mathrm{NO}_{2}\right)$, nitratos $\left(\mathrm{N}-\mathrm{NO}_{3}\right)$, fosfatos $\left(\mathrm{P}-\mathrm{PO}_{4}\right)$ y dureza total (todos en unidades de $\mathrm{mg} / \mathrm{L})$, demanda bioquímica de oxígeno ( $\mathrm{mg} / \mathrm{l} \mathrm{DBO})$, demanda química de oxígeno ( $\mathrm{mg} / \mathrm{L} \mathrm{DQO})$, cloruros $(\mathrm{mg} / \mathrm{l})$, hierro $(\mathrm{mg} / \mathrm{l})$, manganeso $(\mathrm{mg} / \mathrm{L})$ y sulfatos $(\mathrm{mg} / \mathrm{L})$. Se tomó agua en recipientes de vidrio de $250 \mathrm{~mL}$ esterilizados para la medición de coliformes fecales y totales $(\mathrm{NMP} / 100 \mathrm{~mL})$, análisis realizados, de acuerdo con los Standard Methods.

\section{Muestreo e identificación de los macroinvertebrados acuáticos:}

En cada estación, se tomó un tramo de $10 \mathrm{~m}$ de longitud; en este transecto, se instaló la red de pantalla (de $1 \mathrm{~m}$ de ancho) y se removió el lecho de la quebrada arriba de la red, en un trayecto de dos metros, durante 5 minutos; este procedimiento, se repitió 2 veces en diferentes lugares del trayecto. Con la red triangular, se hizo el recorrido del trayecto por el perímetro, lo que permitió recolectar especies en la orilla de la quebrada. Para la recolección manual, se levantaron piedras y se tomaron los macroinvertebrados adheridos a ellas, con pinzas entomológicas; se tomaron paquetes de hojas, los cuales, fueron evaluados sobre una bandeja blanca, para separar los insectos acuáticos. El periodo de captura fue de una hora por estación. Para el muestreo cuantitativo, se empleó una red Surber de $280 \mathrm{~cm}^{2}$ de área. El número de individuos se representó como individuos/tiempo. Se realizó la determinación taxonómica de los macroinvertebrados, con las guías de Roldán (1988), Merrit \& Cummins (1996), Fernández \& Domínguez (2001). Con el listado de familias reportadas, se calculó el índice BMWP/Col (Roldán, 2003).

Análisis estadístico de los datos: Los datos obtenidos en los tres muestreos fueron sometidos a un análisis exploratorio, mediante el uso de programas estadísticos que permiten calcular medidas de tendencia central (media aritmética) y de dispersión (coeficiente de variación relativa de Pearson, C.V). La asociación entre los macroinvertebrados acuáticos y las variables físicas, químicas y microbiológicas, se efectuó mediante un análisis de correlación múltiple. Para determinar si la variación entre los periodos muestreados bajo fenómeno de El Niño y La Niña o entre estaciones de muestreo es significativa, se realizó un anova no paramétrica de una vía (prueba de Kruskal-Wallis). Los análisis estadísticos mencionados anteriormente, se llevaron a cabo con el paquete estadístico Statgraphics centurion XV y Past 2.17.

\section{RESULTADOS Y DISCUSIÓN}

Muestreos 2016: La pluviosidad histórica de la cuenca es de 2304mm; no obstante, durante el 2016, se alcanzó un valor de $1640 \mathrm{~mm}$ (31\% menos); además, se evidencia un cambio de patrón bimodal a multimodal. Durante el 2011, la pluviosidad total fue de 1976 (14\% menos que la media), pero en dos meses (marzo-abril) cayó el 53\% de la precipitación pluvial total del año y un patrón ligeramente monomodal (Figura 2). La reducción en la pluviosidad durante el evento de El Niño estuvo asociada con la variación de las condiciones fisicoquímicas del agua, ya que se relacionó con la disminución en la nitrificación y en la concentración de fósforo total. En contraste, el pH se incrementó ligeramente, al igual que la concentración de nitritos, nitrógeno amoniacal, oxígeno disuelto y la cantidad de coliformes totales. Actualmente, se conoce que la química del agua está relacionada con las perturbaciones que incrementan la precipitación (Blanco et al. 2003).

Las aguas evaluadas en general son oxigenadas, con conductividad eléctrica baja, poco mineralizadas, turbiedad baja, frías, neutras y con concentración de nutrientes baja (Tabla 1). Esta tendencia, se cambia a lo largo del eje longitudinal de las fuentes de agua. Entre los coeficientes de variación, se evidencia que hay variables poco fluctuantes (coeficiente de variación $\mathrm{CV}<30 \%$ ), a nivel espacial y temporal, tales como el $\mathrm{pH}$, la temperatura del agua, la conductividad del agua, sulfatos, sólidos totales, fósforo total soluble, $\mathrm{DBO}_{5}$, nitratos, alcalinidad, dureza total, oxígeno disuelto, $\%$ de saturación de oxígeno y nitratos. Estas variables representan el $52,1 \%$ del grupo evaluado. En un segundo grupo $(100<C V>10)$, se encuentra la velocidad de la corriente, cloruros, color real, hierro total y coliformes totales. Estas variables representan el 23\% del grupo evaluado. Finalmente, las variables con mayor fluctuación fueron el caudal, la turbiedad y la cantidad de Escherichia coli, que representan el 13\% de las variables estudiadas.

$\mathrm{Al}$ evaluar la significancia estadística de los cambios en las variables fisicoquímicas entre un ciclo climático influenciado por La Niña y uno por El Ñiño, las variables físicas no evidenciaron cambios, excepto la cantidad de sólidos totales presentes en el agua. En contraste, variables químicas, como la concentración de oxígeno disuelto, el $\mathrm{pH}$, la dureza, la concentración de nitritos, nitratos, fósforo total y nitrógeno amoniacal, señalaron cambios estadísticos significativos. Blanco et al. (2003) encontraron que los ENOS en un río se traducen, en su orden, en una reducción de caudales mínimos 


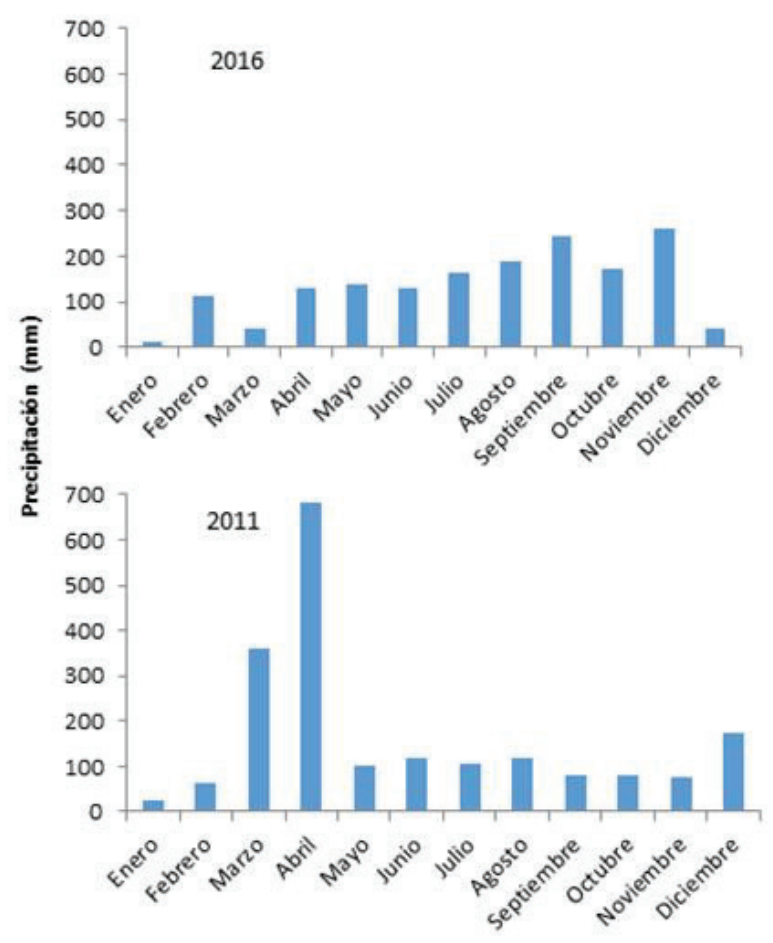

Figura 2. Registro de precipitaciones 2016 (El Niño) y 2011 (La Niña).

Tabla 1. Medidas de tendencia central y de dispersión de las variables fisicoquímicas y microbiológicas.

\begin{tabular}{|c|c|c|c|c|c|}
\hline Variable & Promedio & Valor máximo & Valor mínimo & Desviación estándar & $\mathrm{CV}$ \\
\hline Oxígeno disuelto $\left(\mathrm{mg} / \mathrm{l} \mathrm{O}_{2}\right)$ & 6,86 & 8,46 & 1,30 & 1,85 & 26,9 \\
\hline Temperatura del agua $\left({ }^{\circ} \mathrm{C}\right)$ & 17,68 & 22,30 & 15,00 & 2,70 & 15,3 \\
\hline Porcentaje de saturación de oxígeno (\%) & 71,08 & 85,20 & 14,60 & 17,19 & 24,2 \\
\hline Caudal (L/s) & 253,32 & 1071,90 & 30,34 & 293,28 & 115,8 \\
\hline Velocidad (m/s) & 59,72 & 134,53 & 5,95 & 32,45 & 54,3 \\
\hline $\mathrm{pH}$ Unidades de $\mathrm{pH}$ ) & 7,16 & 7,35 & 7,00 & 0,13 & 1,9 \\
\hline Turbiedad (U.N.T) & 1,48 & 4,86 & 0,40 & 1,53 & 103,0 \\
\hline Conductividad eléctrica $(\mu \mathrm{S} / \mathrm{cm})$ & 31,32 & 43,90 & 26,40 & 5,64 & 18,0 \\
\hline Alcalinidad (mg/L CaCO 3$)$ & 15,35 & 18,88 & 10,18 & 3,47 & 22,6 \\
\hline Dureza total (mg/L CaCO 3$)$ & 13,43 & 18,13 & 9,25 & 2,87 & 21,4 \\
\hline Cloruros (mg/L Cl- ) & 3,17 & 5,50 & 1,00 & 1,88 & 59,2 \\
\hline Color real (U.C) & 12,42 & 25,79 & 3,59 & 8,27 & 66,6 \\
\hline Nitritos (mg/L NO 2$)$ & 0,00 & 0,00 & 0,00 & 0,00 & 28,8 \\
\hline Sulfatos (mg/L SO 4$)$ & 2,03 & 2,30 & 2,00 & 0,10 & 4,9 \\
\hline Hierro total (mg/L Fe) & 0,39 & 1,11 & 0,10 & 0,35 & 89,5 \\
\hline Nitratos (mg/L NO $3-)$ & 0,12 & 0,17 & 0,10 & 0,03 & 24,1 \\
\hline Fósforo total soluble (mg/L -P) & 0,10 & 0,10 & 0,10 & 0,00 & 0,0 \\
\hline Nitrógeno amoniacal (mg/L NH3-N) & 0,40 & 0,40 & 0,40 & 0,00 & 0,0 \\
\hline Demanda Química de Oxígeno (mg/L) & 11,19 & 15,00 & 10,00 & 1,91 & 17,1 \\
\hline Demanda Bioquímica de Oxígeno (mg/L) & 4,00 & 4,00 & 4,00 & 0,00 & 0,00 \\
\hline Sólidos totales $(\mathrm{mg} / \mathrm{L})$ & 38,94 & 53,30 & 20,00 & 10,02 & 25,73 \\
\hline Coliformes totales (U.F.C./100 mL) & 4572,22 & 8200,00 & 1400,00 & 2277,21 & 49,81 \\
\hline Escherichia coli (U.F.C./100 mL) & 361,11 & 1300,00 & 50,00 & 403,72 & 111,80 \\
\hline
\end{tabular}


e incremento de caudales máximos. Tales cambios, se reflejan en variaciones de las concentraciones de iones (fosfatos, nitratos, hierro), influenciados por la magnitud de la escorrentía máxima.

Se encontraron 43 familias de macroinvertebrados acuáticos entre los que predominaron los moluscos (Planorbidae y Lymnaeidae), tricópteros (Glossosomatidae y Hydropsychidae), hemípteros (Veliidae y Gerridae), odonata (Coenagrionidae) y el nematodo (Chordodidae). El índice BMWP/col presentó correlaciones significativas con casi todas las variables, menos con la velocidad de la corriente y la época del muestreo, lo que indica que no fue sensible a las variaciones del caudal $(\mathrm{r}=-0,31, \mathrm{p}=0,08)$ ni a la fecha en que se realizó la investigación $(r=-0,33, p=0,093)$. El anova presenta diferencias entre las estaciones de muestreo, más no entre las diferentes épocas de muestreo, lo que indicaría que las condiciones del agua fueron poco fluctuantes estadísticamente (Tabla 2 año 2016). Respecto a la calidad biológica del agua, esta fue óptima en la zona de las bocatomas, pero en la medida que se avanza longitudinalmente, disminuyen los valores del índice BMWP/ Col al acercarse al casco urbano (Figura 2).

Tabla 2. Análisis de varianza de las variables estudiadas en las seis estaciones de muestreo comparando 2011-2016.

\begin{tabular}{|c|c|c|c|c|c|}
\hline \multicolumn{2}{|l|}{ Año } & \multicolumn{2}{|c|}{2016} & \multicolumn{2}{|c|}{ 2006-2011 } \\
\hline Variable & Factor & Valor F & Valor $\mathrm{p}$ & Valor F & Valor p \\
\hline \multirow{2}{*}{ Índice BMWP/Col } & Estaciones & 3,9 & 0,031 & 5,16 & 0,021 \\
\hline & Muestreos & 2,3 & 0,147 & 13,3 & 0,0026 \\
\hline \multirow{2}{*}{ Riqueza de familias } & Estaciones & 1,81 & 0,198 & 0,67 & 0,5278 \\
\hline & Muestreos & 2,99 & 0,096 & 0,8 & 0,3449 \\
\hline \multirow{2}{*}{ Índice de diversidad } & Estaciones & 14,26 & 0,003 & 2,3 & 0,1365 \\
\hline & Muestreos & 3,9 & 0,0559 & 0 & 0,9465 \\
\hline \multirow{2}{*}{ Índice de equidad } & Estaciones & 11,71 & 0,0006 & 2,65 & 0,1055 \\
\hline & Muestreos & 0,44 & 0,653 & 1,76 & 0,2052 \\
\hline \multirow{2}{*}{ Índice de dominancia } & Estaciones & 9,24 & 0,0016 & 1,56 & 0,237 \\
\hline & Muestreos & 1,17 & 0,3481 & 0,04 & 0,8406 \\
\hline \multirow{2}{*}{ Oxígeno disuelto } & Estaciones & 6,95 & 0,0048 & 1,01 & 0,3896 \\
\hline & Muestreos & 2,97 & 0,0967 & 21,37 & 0,0004 \\
\hline \multirow{2}{*}{ Temperatura del agua } & Estaciones & 125,03 & 0 & 2,54 & 0,1143 \\
\hline & Muestreos & 3,15 & 0,082 & 1,13 & 0,3059 \\
\hline \multirow{2}{*}{ Porcentaje de saturación de OD } & Estaciones & 4,16 & 0,0264 & 6,27 & 0,0114 \\
\hline & Muestreos & 2,59 & 0,123 & 0,44 & 0,518 \\
\hline \multirow{2}{*}{ Caudal } & Estaciones & 10,96 & 0,0008 & 0,58 & 0,572 \\
\hline & Muestreos & 6,54 & 0,6001 & 1,88 & 0,1924 \\
\hline \multirow{2}{*}{$\mathrm{pH}$} & Estaciones & 6,84 & 0,005 & 1,55 & 0,246 \\
\hline & Muestreos & 0,18 & 0,8396 & 5,07 & 0 \\
\hline \multirow{2}{*}{ Conductividad eléctrica } & Estaciones & 1,9 & 0,1189 & 3,15 & 0,0742 \\
\hline & Muestreos & 7,87 & 0,8765 & 0,12 & 0,7292 \\
\hline \multirow{2}{*}{ Dureza } & Estaciones & 2,45 & 0,3216 & 0,69 & 0,5171 \\
\hline & Muestreos & 2,87 & 0,1236 & 11,85 & 0,004 \\
\hline \multirow{2}{*}{ Nitritos } & Estaciones & 19,5 & 0,0012 & 0,72 & 0,5017 \\
\hline & Muestreos & 1,96 & 0,6312 & 104,9 & 0 \\
\hline \multirow{2}{*}{ Nitratos } & Estaciones & 16,3 & 0,003 & 4,25 & 0,3167 \\
\hline & Muestreos & 3,1 & 0,0678 & 37,56 & 0 \\
\hline \multirow{2}{*}{ Fósforo total } & Estaciones & 21 & 0,004 & 1,3 & 0,3927 \\
\hline & Muestreos & 1,9 & 0,4356 & 20 & 0,0005 \\
\hline \multirow{2}{*}{ Nitrógeno amoniacal } & Estaciones & 23,6 & 0,0005 & 0,37 & 0,6983 \\
\hline & Muestreos & 1,9 & 0,4568 & 62,26 & 0 \\
\hline \multirow{2}{*}{ sólidos totales } & Estaciones & 3,21 & 0,3741 & 0,88 & 0,4402 \\
\hline & Muestreos & 3,56 & 0,1121 & 42,54 & 0 \\
\hline \multirow{2}{*}{ Coliformes Totales } & Estaciones & 4,54 & 0,034 & 0,06 & 0,9404 \\
\hline & Muestreos & 2,45 & 0,0823 & 22,01 & 0,0003 \\
\hline
\end{tabular}


Comparación ciclo 2011 (La Niña) contra ciclo 2016 (E1 Niño): Sobre el efecto del fenómeno de El Niño, el análisis de varianza con comparación de medias realizadas por el test de Tukey, permitió identificar que, entre los muestreos de 2011 y de 2016, para la mayoría de las variables evaluadas, hay diferencias, no así entre las estaciones (Tabla 2 comparación 2011-2016).

Se graficaron las variables que presentaron diferencias estadísticas entre los muestreos de 2011, realizados por García et al. 2012 (ciclo influenciado por La Niña) y los actuales de 2016 (ciclo influenciado por El Niño), según la clasificación de Null (2017). Para este análisis gráfico, se tomó en cuenta solo los datos de las estaciones ubicadas sobre las bocatomas (E1, E3 y E4), ya que fue posible obtener toda la información fisicoquímica y microbiológica (Figura 3). Se observa en las gráficas que, en general, se separan los resultados de los muestreos influenciados por el ciclo de La Niña (2011) de los influenciados por el ciclo de El Niño (2016). Las estaciones monitoreadas presentan aguas oxigenadas, con concentraciones superiores a los $5 \mathrm{mg} / \mathrm{l}$, valores que son mayores en los muestreos influenciados por el ciclo de El Niño, debido a un menor nivel de precipitaciones y de caudal, los cuales, arrastran material orgánico alóctono que demanda oxígeno. El pH registró valores circumneutrales, con valores superiores a 7, durante El Niño y valores ligeramente ácidos, entre 6,2 a 6,9, durante el ciclo de La Niña, debido a una mayor mineralización de la materia orgánica y a la reducción del flujo de agua.
El patrón de variación de la temperatura del agua fue fluctuante, aunque se evidenció que la estación E1 (bocatoma quebrada La Cimarronas) presentó mayor temperatura durante El Niño y las otras estaciones E3 y E4 (La Madera y Andes), durante La Niña, lo cual, se pudo deber a que la E1 fue la primera estación muestreada y a esa hora es más fresco el día, especialmente, en época seca. No se encontró diferencias estadísticas en la temperatura del agua ni en el caudal de las fuentes hídricas, pese a que se observó una reducción considerable del flujo de agua en las fuentes hídricas. Roldán \& Ramírez (2008) afirman que las aguas tropicales se caracterizan por conservar una temperatura más o menos constante a lo largo del año.

Los sólidos totales presentan mayores valores durante La Niña, debido al aumento de las precipitaciones y arrastre de materiales. Esta misma tendencia la evidenció la dureza del agua, aunque los valores registrados en todos los muestreos son bajos, lo que indica aguas blandas.

Las formas de nitrógeno en el agua fueron fluctuantes, ya que se evidencia que la concentración de nitritos y de nitrógeno en forma amoniacal fueron muy bajas y que durante El Niño se presentaron los mayores valores, lo que puede reflejar un proceso de nitrificación mayor en esta fase climática. En contraste, las concentraciones de nitratos fueron superiores en el ciclo climático influenciado por $\mathrm{La}$ Niña, lo que indicaría que, con el incremento de las lluvias, reducción de la temperatura y aumento de la escorrentía, predominaron
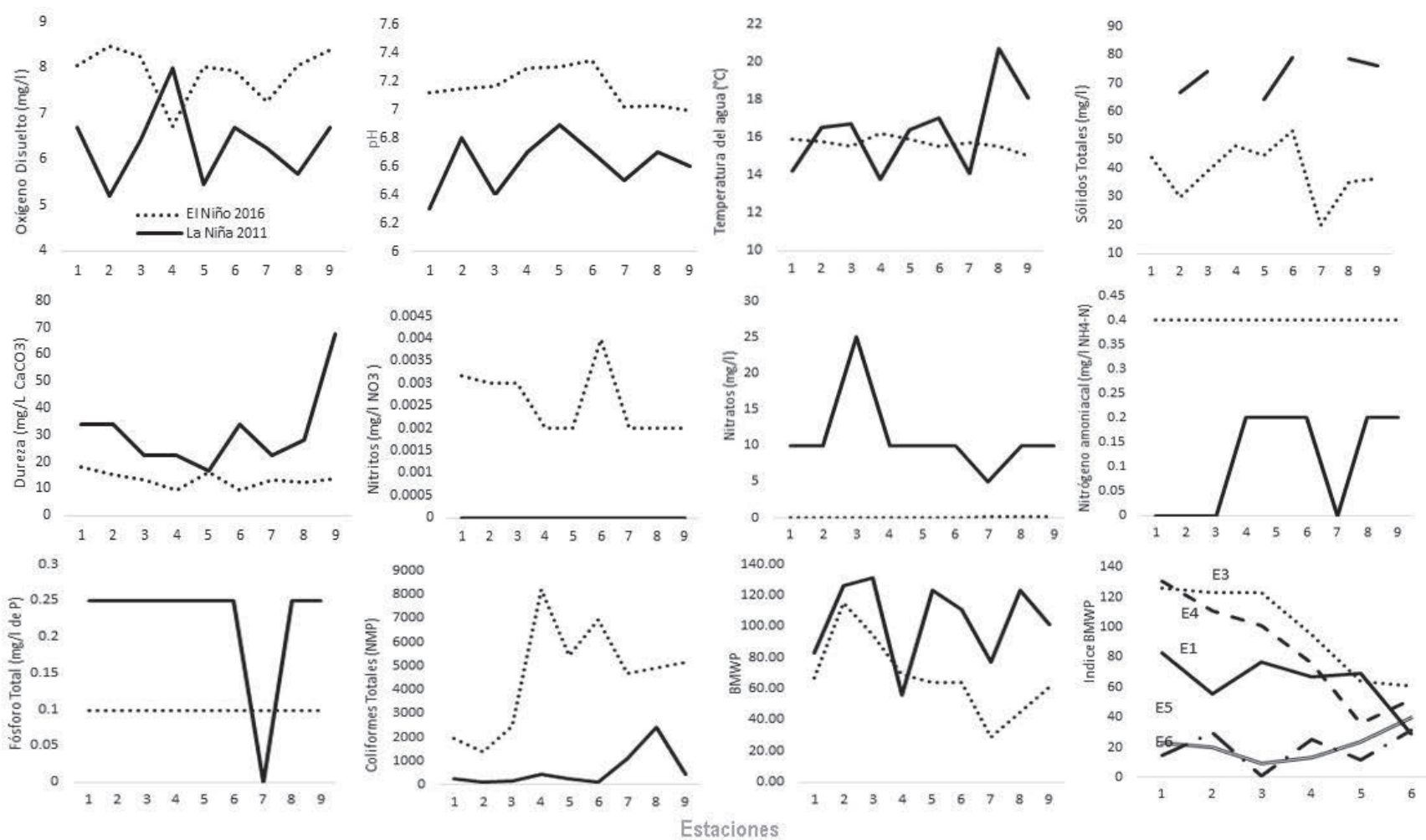

Figura 3. Registro de las variables que presentaron diferencias con $\mathrm{p}<0,05$ entre el ciclo climático influenciado por La Niña (2011) y por El Niño (2016). 
los nitratos, como la fuente de nitrógeno para los organismos. La concentración de fósforo total fue mayor en general durante La Niña, lo cual, se pudo deber a flujos asociados a procesos de escorrentía y arrastre. La cantidad de coliformes totales fue muy superior durante El Niño, lo que se pudo deber a la reducción del volumen de agua que diluye microorganismos provenientes de potreros, hacia las fuentes hídricas y fuentes puntuales. Además, que durante La Niña, la mayor radiación solar incide negativamente en la proliferación de los coliformes.

Los mayores valores del índice BMWP, se registraron durante La Niña, lo que pudo estar asociado al incremento del perímetro húmedo, además del aporte en riqueza de especies, debido a los procesos de colonización de nuevos sustratos, los cuales, contribuyen a incrementar el valor del índice BMWP. Este índice presentó una respuesta acoplada a la concentración de coliformes totales, lo que implica que estas variables dan información diferente y complementaria, ya que se identifica una buena calidad biológica del agua durante La Niña (excepto en la bocatoma de la quebrada La Cimarronas en el muestreo 2), la cual, disminuye durante El Niño. $\mathrm{Y}$ respecto a la calidad microbiológica del agua, se evidencia una mejor calidad durante La Niña que durante El Niño. Se evidencia el deterioro de la calidad del agua, especialmente, en los tramos bajos de la quebrada La Cimarronas, donde se observa una sustitución de los taxones característicos de aguas limpias, como Polythoridae, Ptilodactylidae, Psephenidae, Odontoceridae, Leptophlebiidae, por un grupo de taxones más tolerantes, como Chironomidae, Baetidae, Simuliidae, Physidae, Hydrophilidae, Tipulidae, Naididae; esta tendencia, se encuentra asociada a la polución del agua y a los cambios en los usos del suelo. Este patrón no ha cambiado (García et al. 2012), lo que evidencia que desde las entidades del orden municipal y regional no se han realizado acciones efectivas para mejorar la calidad del agua que llega a las bocatomas y que surte al acueducto de El Carmen de Viboral.

Se evidencia que las estaciones ubicadas en la parte alta de la cuenca (bocatomas) tienden a disminuir la calidad biológica del agua entre el ciclo de La Niña y El Niño; en contraste, las estaciones, ubicadas en la parte baja de la cuenca, tienen los mayores problemas de contaminación y los bajos valores del BMWP tienden a incrementar estos al pasar del ciclo influenciado por La Niña al influenciado por El Niño. Entre las microcuencas, se identifica que la quebrada La Cimarronas es la que presenta mayores perturbaciones (agricultura, ganadería, cultivo de flores, deforestación, asentamientos humanos), lo que incide en el menor valor del índice BMWP/Col, mayor concentración de coliformes totales y fecales y menores valores de los índices comunitarios, que se documenta en varios trabajos (Montoya, 2007; García et al. 2012) y en la presente investigación. Entre las causas que generan baja diversidad en las estaciones E5 y E6, se encuentra, principalmente, la contaminación en estas zonas y los tipos de sustratos hallados, ya que estos son primariamente arenosos de aluvión y arcilla, característicos de las partes bajas de las quebradas; estos tipos de sustratos son muy pobres en fauna béntica, ya que son un medio muy inestable para su establecimiento (Roldán \& Ramírez, 2008). La calidad del agua en las zonas de bocatomas, aunque fluctúo, no puso en riesgo a la población, aunque en 2016, se alcanzó a presentar racionamiento del recurso, debido al bajo nivel en las fuentes.

La concentración de coliformes totales reflejó cambios estadísticos entre el ciclo climático influenciado por La Niña y por El Niño. Emiliani (2004) registró que los patrones temporales de concentración de E. coli en un curso secundario $\left(\mathrm{Q}=101 \mathrm{~m}^{3} / \mathrm{s}\right)$ siguieron a los del río Paraná, pero las concentraciones y los picos de E. coli producidos por lluvias excepcionales, mostraron una magnitud mayor.

Los índices comunitarios presentaron diferencias significativas a nivel espacial, pero no a nivel temporal, ni entre los periodos climáticos de La Niña y El Niño. En contraste, el índice BMWP/ Col fue un buen indicador de la calidad biológica del agua, ya que evidenció cambios entre sitios de muestreo y entre periodos climáticos, convirtiéndose en la única variable biológica, que sirvió para señalar la variabilidad de las condiciones de las fuentes hídricas. Esto, se pudo deber a que al ser un índice que refleja puntajes sobre la presencia de una familia de insectos acuáticos puede detectar rápidamente variaciones en las condiciones ambientales de las fuentes hídricas. Roldán-Pérez (2016) considera que no es extraño este tipo de relación en los macroinvertebrados acuáticos, ya que, debido al ambiente dinámico en que habitan con disturbios frecuentes e impredecibles, los organismos tienen, generalmente, altas tasas de dispersión y deriva, recolonizando, de esta manera, los hábitats desnudos (Montoya, 2007; Longo-Sánchez et al. 2009; Mosquera-Murillo \& Mosquera-Mosquera, 2017).

Las fuentes hídricas abastecedoras del área urbana del municipio de El Carmen de Viboral presentan una aceptable calidad biológica y fisicoquímica. Según el Decreto 1594 de 1989, Capítulo IV, las bocatomas actuales (E1, E3 y E4) y la futura (E2) no tienen restricciones para ser usadas como fuente de aguas para potabilizar. Se hace necesaria una mayor intervención en la zona del nacimiento de la quebrada La Cimarronas, ya que esta fuente presenta las menores condiciones de calidad del agua, respecto a las otras microcuencas.

Durante el ciclo afectado por El Niño, se presentó mayor concentración de oxígeno disuelto, $\mathrm{pH}$ circumneutral, menor temperatura del agua y mayores concentraciones de nitritos, nitrógeno amoniacal y coliformes totales. Durante el ciclo afectado por La Niña, se manifestó mayor temperatura del agua, sólidos totales, dureza, nitratos, fósforo total y mejores condiciones de calidad biológica del agua según el índice BMWP. La evaluación sincrónica con fenómenos climáticos es compleja, ya que pequeñas variaciones en la pluviosidad diaria generan variaciones adicionales a las estacionales.

Conflictos de intereses: El manuscrito fue preparado y revisado con la participación de todos los autores, quienes declaramos que no existe ningún conflicto de intereses que ponga en riesgo la validez de los resultados presentados. 


\section{REFERENCIAS}

1. AGUIRRE-CÁRDENAS, A.; ALMANZA-VELAZCO, S.; IVANOVA, Y. 2018. Influencia del fenómeno de oscilación cuasi-bienal en las afluencias del sistema sur de abastecimiento de Bogotá. Rev. Ingeniería, Investigación y Desarrollo.18(1):16-24. https://doi. org/10.19053/1900771X.v18.n1.2018.7822

2. ÁVILA DIAZ, A.; CARVAJAL ESCOBAR, Y.; GUTIERREZ SERNA, S. 2014. Análisis de la influencia de El Niño y La Niña en la oferta hídrica mensual de la cuenca del río Cali. Tecnura. 18(41):120-133. https://doi.org/10.14483/ udistrital.jour.tecnura.2014.3.a09

3. BLANCO, J.; VÁSQUEZ, G.; RAMÍREZ, J.; NAVARRETE, A.M. 2003. Variación de algunos parámetros fisicoquímicos en el río Pescador, Valle del Cauca, durante el ciclo El Niño 1997/1998-La Niña 1998/1999. Actual. Biol. 25(78):59-69.

4. CHILDRESS, V. 2015. Water, conflict, and technology. Technology \& Engineering Teacher. 74(4):22-27.

5. COMISIÓN EUROPEA. 2000. La Directiva Marco del Agua de la UE. El agua de Europa está sometida a presiones. Disponible desde Internet en: http://ec.europa.eu/ environment/pubs/pdf/factsheets/wfd/es.pdf (con acceso el 12/06/2018).

6. CORPORACIÓN AUTÓNOMA REGIONAL DE LAS CUENCAS DE LOS RIOS NEGRO Y NARE, CORNARE. 2006. Plan de Ordenación y Manejo de la Cuenca La Cimarronas, Municipio de El Carmen de Viboral Disponible desde Internet en: http://www.cornare.gov.co/ POMCAS/Documentos/Cimarrona.pdf (con acceso el 12/06/2018).

7. EMILIANI, F. 2004. Effects of hydroclimatic anomalies on bacteriological quality of the Middle Paraná River (Santa Fe, Argentina). Rev. Argent. Microbiol. 36(4):193-201.

8. FERNÁNDEZ, H.R.; DOMÍNGUEZ, E. 2001. Guía para la determinación de los artrópodos bentónicos sudamericanos. Ed. Universitaria de Tucumán (Argentina). 282p.

9. GARCÍA, J.; CARDONA, J.; MONTOYA, Y. 2012. Caracterización de la calidad del agua de la parte alta de la microcuenca de la quebrada la Cimarronas, El Carmen de Viboral (Antioquia), utilizando macroinvertebrados acuáticos. Investigación, Biodiversidad y Desarrollo. 31(1):5-18. https://doi.org/10.18636/ribd.v31i1.269

10. NULL, J. 2017. El Niño and La Niña Years and Intensities. Disponible desde Internet en: http://ggweather.com/ enso/oni.htm. (con acceso el 8/04/2018).
11. INSTITUTO DE HIDROLOGÍA, METEOROLOGÍA Y ESTUDIOS AMBIENTALES, IDEAM. 2015. Estudio nacional del agua. Disponible en: https://www. minambiente.gov.co/index.php/noticias/123-noticiaseducacion-ambiental/1960-estudio-nacional-del-agua (con acceso el 4/02/2018).

12. LONGO-SÁNCHEZ, M.C.; GÓMEZ-AGUIRRE, A.M.; BLANCO, J.F.; ZAMORA-GONZÁLEZ, H. 2009. Cambios multianuales y espaciales de la composición y estructura del ensamblaje de insectos acuáticos en las quebradas perennes de la isla Gorgona, Col. Actual. Biol. 31:141-160.

13. MERRITT, R.W.; CUMMINS, K.W. 1996. An introduction to the aquatic insects of North America. Kendall/Hunt Publishing Cc (USA). 754p.

14. MONTOYA, Y. 2007. Colonización de sustratos rocosos por los macroinvertebrados acuáticos en la quebrada Los Andes, El Carmen de Viboral, Antioquia-Colombia. Rev. UCO. 23(1):89-104.

15. MOSQUERA-MURILLO, Z.; MOSQUERA-MOSQUERA, M.M. 2017. Diversidad de la entomofauna acuática y calidad de agua en quebradas del río San Juan, Chocó Colombia. Rev. U.D.C.A Act. \& Div. Cient. 20(1):149-161. https://doi.org/10.31910/rudca.v20.n1.2017.72

16. POVEDA, G. 2004. La hidroclimatología de Colombia: una síntesis desde la escala inter-decadal hasta la escala diurna. Rev. Acad. Col. Cienc. 28(107):201-222.

17. PUERTA, O.; CARVAJAL, Y. 2008. Incidencia de El NiñoOscilación del Sur en la precipitación y la temperatura del aire en Colombia. Rev. Cient. Ing. y Des. 23:104-118.

18. RAMÍREZ-BUÍLES, V.H.; JARAMILLO-ROBLEDO, A. 2009. Relación entre el Índice oceánico de El Niño y la lluvia, en la región andina central de Colombia. Cenicafé. 60(2):161172.

19. RAMÍREZ, A.; GUTIÉRREZ-FONSECA, P. 2014. Estudios sobre macroinvertebrados acuáticos en América Latina: avances recientes y direcciones futuras. Rev. Biologia Tropical. 62(suppl 2):9-20. https://doi.org/10.15517/rbt. v62i0.15775

20. ROCHA-FELICES, A. 2002. El fenómeno el niño de 1578 y el pago de impuestos. Rev. Ing. Civil Coleg de Ingen. del Perú-Lima. 6(28):1-20.

21. ROLDÁN, G. 2016. Los macroinvertebrados como bioindicadores de la calidad del agua: cuatro décadas de desarrollo en Colombia y Latinoamérica. Rev. Acad. 
Col. Cienc. Ex. Fis. Nat. 40(155):254-274. http://dx.doi. org $/ 10.18257 /$ raccefyn.335

22. ROLDÁN, G.; RAMÍREZ, J. 2008. Fundamentos de Limnología, Editorial Universidad Antioquia; Antioquia (Colombia). 440p.
23. ROLDÁN, G. 2003. Bioindicación de la calidad del agua en Colombia. Universidad de Antioquia. Medellín (Colombia). $170 \mathrm{p}$.

24. ROLDÁN, G. 1988. Guía para el estudio de Macroinvertebrados Acuáticos del Departamento de Antioquia. Presencia Ltda (Colombia). 216p. 